\title{
Integrated analyses of microRNA and mRNA expression profiles in aggressive papillary thyroid carcinoma
}

\author{
ZHILI YANG ${ }^{*}$, ZIMING YUAN*, YOUBEN FAN, XIANZHAO DENG and QI ZHENG \\ Department of Surgery, The Sixth People's Hospital Affiliated to Shanghai Jiao Tong University, Shanghai 200233, P.R. China
}

Received March 20, 2013; Accepted August 19, 2013

DOI: $10.3892 / \mathrm{mmr} .2013 .1699$

\begin{abstract}
RNAs (miRNAs) are involved in the pathogenesis of diverse human cancers through its target genes, including papillary thyroid cancer (PTC). However, there are few studies regarding associations between clinicopathological features of PTC with the expression of specific miRNAs and its potential target genes. In the present study, analysis of miRNA was integrated with mRNA expression profiles in aggressive PTC. miRNA and gene expression arrays were used to identify a subset of differentially expressed miRNAs and mRNAs between aggressive and non-aggressive PTCs. These miRNAs and mRNAs were further validated by qPCR in a cohort of 20 PTCs with extrathyroidal invasion and/ or distant metastases, and 20 PTCs with no extrathyroidal invasion. The target of these miRNAs was determined by luciferase reporter and bioinformatic analysis. The miRNA arrays identified 14 upregulated miRNAs and 10 downregulated miRNAs in aggressive compared with non-aggressive PTCs. Significant miRNA deregulation was confirmed in the validation cohort, with upregulation of miR-146b-5p and miR-221/222 and downregulation of miR-16 and miR-613 in aggressive PTCs. The gene arrays identified 2000 differentially expressed genes, in which TIMP3, ZNFR3, FN1 and ITGA2 were observed to be target genes inversely correlated with miR-221/222, miR-146b-5p, miR-613 and miR-16, respectively. The results of the present study indicated the potential importance of miR-221/222, miR-146b-5p, miR-16 and miR-613 in determining the aggressive properties of PTC by targeting TIMP3, ZNFR3, FN1 and ITGA2, respectively. Additional studies should be conducted to confirm the results.
\end{abstract}

Correspondence to: Professor Qi Zheng, Department of Surgery, The Sixth People's Hospital Affiliated to Shanghai Jiao Tong University, 600 Yishan Road, Shanghai 200233, P.R. China

E-mail: zhengqi1957@yahoo.com.cn

*Contributed equally

Key words: microRNA, mRNA, papillary thyroid carcinoma, aggressiveness

\section{Introduction}

Papillary thyroid carcinoma (PTC) is the most common type of well-differentiated cancer of the thyroid. It represents $\sim 80-85 \%$ of well-differentiated thyroid cancers (1). However, certain cases show relatively early recurrence, severe invasion, multiple lymph node metastasis or distant metastasis (2). Therefore, it is important to identify the characteristics of PTC with a high risk of invasion and metastasis.

microRNAs (miRNAs) are endogenous single- stranded non-coding RNAs that bind to the 3 ' non-coding region of the target mRNAs, resulting in their selective degradation or inhibition of translation. Therefore, by regulating their target genes, miRNAs are known to be involved in a wide range of biological functions, such as invasion, metastasis, proliferation and differentiation $(3,4)$. Deregulation of miRNA expression has been demonstrated in thyroid carcinoma (5). In PTC, it has been suggested that miRNA expression provides an indication of the aggressiveness of the cancer (6).

mRNA expression profiling has determined that transcriptional abnormalities of numerous genes are responsible for the invasion of PTC (7). In a previous study, it was shown that amyloid precursor protein expression was correlated with large tumor size, extracapsular invasion and lymph node metastasis in PTC (8).

In the present study, microarrays were used to measure the expression levels of miRNAs and mRNAs simultaneously in non-aggressive and aggressive PTCs, to investigate the potential involvement of miRNAs in invasion and metastasis. The analyses were integrated and extensive in the search for possible candidate miRNAs and their mRNA targets for use in further investigations and clinical applications.

\section{Materials and methods}

Clinical sample collection. The aggressive and non-aggressive PTC tissues used in this study were obtained from the Sixth People's Hospital Affiliated to Shanghai Jiao Tong University (Shanghai, China). The aggressive tumors were identified based on the presence of extrathyroidal extension and/or lymph node and distant metastases. In the non-aggressive group, samples were selected with no extrathyroidal dissemination or regional lymphatic and distant metastases. Specimens were snap-frozen in liquid nitrogen. Histopathology of the tissues was diagnosed by one pathologist. The collection and 
use of the patient samples was reviewed and approved by the ethics committee of the Sixth People's Hospital Affiliated to Shanghai Jiao Tong University, and written informed consent was obtained from the patients.

Total RNA isolation. Total RNA was isolated from the aggressive and non-aggressive PTC tissues using TRIzol reagent (Invitrogen Life Technologies, Carlsbad, CA, USA), according to the manufacturer's instructions. Total RNA quantification was performed using the ND-1000 spectrophotometer (Nanodrop; Thermo Scientific Wilmington, DE, USA). RNA integrity and the content of miRNAs was analyzed by capillary electrophoresis using the Agilent Bioanalyzer 2100 (Agilent Technologies, Santa Clara, CA, USA).

miRNA and gene expression arrays. miRNA expression profiles were conducted in the aggressive and non-aggressive PTC tissues. Analyses were performed using the Affymetrix ${ }^{\circledR}$ GeneChip miRNA 2.0 array (Affymetrix, Santa Clara, CA, USA), that allows the detection of 1105 known human miRNAs (miRBase v.15; Affymetrix). Total RNA (200 ng) was labeled with FlashTag Biotin HSR, according to the Affymetrix instructions. The miRNA Array library file package was downloaded and installed into Affymetrix GeneChip Command Console software. Labeled samples were hybridized with the arrays and then washed with PBS, stained and scanned according to the Affymetrix instructions. The miRNA QC Tool software (Affymetrix) was used for data summarization, normalization and quality control.

Gene expression profiling was conducted in the aggressive and non-aggressive PTC tissues, previously analyzed for miRNA profiling. The GeneChip ${ }^{\circledR}$ Human Gene 1.0 ST Array (Affymetrix), consisting of 764,885 oligonucleotide probes that span conserved exons across the transcripts of the targeted full-length genes, was used. Total RNA (800 ng) was labeled using the Affymetrix GeneChip WT terminal labeling kit according to the manufacturer's instructions. A total of $1.65 \mathrm{mg}$ labeled cRNA was used to prepare the hybridization samples and the hybridization was conducted at $65^{\circ} \mathrm{C}$ for $17 \mathrm{~h}$ in a Hybridization Oven Rotator (Affymetrix). The arrays were washed and Stabilization and Drying solution (Affymetrix) was used according to the manufacturer's instructions. Slides were scanned on an Affymetrix microarray scanner and Affymetrix GeneChip analysis software was used for imaging analysis.

Identification of miRNA target genes. The conventional online programs, including miRanda (http://www.microrna.org), Targetscan (http://www.targetscan.org) and Findtar (http:// bio.sz.tsinghua.edu.cn), were used to predict the targets of the miRNAs. The targets predicted by the three programs were further analyzed. To identify the most likely targets, the mRNA and miRNA expression data obtained on the same biological samples using Microsoft Excel tool were integrated. Two-fold upregulated miRNA and corresponding 2-fold downregulated mRNA target were selected for further investigation.

Validation of miRNA and $m R N A$ expression levels with qPCR. Total RNA was prepared from tissues using the RNeasy extraction kit (GE Healthcare, Pittsburgh, PA, USA) and reverse transcribed using High-capacity cDNA Reverse Transcription kits (Applied Biosystems, Victoria, Australia) according to the manufacturer's instructions. qPCR was performed on a 7300 Fast Real-Time PCR system (Applied Biosystems, Foster City, CA, USA) using SYBR-Green PCR Master mix (Applied Biosystems).

miRNA expression analysis was validated using the MicroRNA Assay kit (Applied Biosystems, Foster City, CA, USA), which incorporates a target-specific stem-loop reverse transcription primer to provide specificity for the mature miRNA target. Small nucleolar RNA RNU44 served as an endogenous control for the normalization of RNA input. Specific primers of mRNA expression analysis and the endogenous control were provided by Invitrogen Life Technologies (Shanghai, China). The specificity of the PCR products was tested by dissociation curves. Relative values of transcripts were calculated using the equation, $2^{-\Delta \Delta \mathrm{Ct}}$, where $\Delta \mathrm{Ct}$ is equal to the difference in threshold cycles for target and reference genes. Each experiment was performed in triplicate.

Luciferase reporter assays. Luciferase reporter vectors containing the 3'-UTR of target TIMP3, ZNFR3, FN1 and $I T G A 2$, were generated following PCR amplification of human cDNA and cloned into the pmirGLO Dual-Luciferase miRNA target expression vector (Promega, Madison, WI, USA), immediately downstream from the stop codon of the luciferase gene. The sequence of each insert was confirmed by sequencing. A549 cells were plated in 24-well plates and $24 \mathrm{~h}$ later cotransfected with $50 \mathrm{ng}$ pmirGLO dual-luciferase constructs, containing the indicated 3'-UTRs of target genes, and with $32 \mathrm{mM}$ pre-miR ${ }^{\mathrm{TM}}$ miRNA Precursor MoleculesNegative Control or pre-miR miRNA using Lipofectamine 2000 (Life Technologies, Carlsbad, CA, USA). Lysates were collected $24 \mathrm{~h}$ following transfection and Firefly and Renilla luciferase activities were consecutively measured by the Dual-Luciferase Reporter assay (Promega) according to the manufacturer's instructions. Relative luciferase activity was calculated by normalizing the ratio of Firefly/Renilla luciferase to that of negative control-transfected cells. Transfections were performed in triplicate.

Statistical analysis. Differences in miRNA and mRNA expression levels between groups were analyzed with a t-test. Data are presented as means \pm SE. Statistical analyses were performed using SPSS software (version 10.0; SPSS, Inc., Chicago, IL, USA). P<0.05 was considered to indicate a statistically significant difference. Agglomerative hierarchical clustering analysis was performed using Cluster software and Treeview software (Eisen Lab, Berkeley, CA, USA).

\section{Results}

miRNA expression assessed with miRNA array. miRNA expression was initially assessed with miRNA array in 6 snap-frozen PTC tissues obtained from 3 aggressive tumors and 3 non-aggressive tumors. The aggressive tumors were identified based on the presence of extrathyroidal extension and/or lymph node metastases. miRNA array analysis for the expression of 1105 miRNAs demonstrated a subset of upregulated miRNAs (miR-146b-5p, miR-221, miR-222, miR-210, miR-214, miR-1244, miR-134, miR-127-3p, miR-130b, 
Table I. Clinicopathogenetical features of PTC samples.

\begin{tabular}{lcc}
\hline $\begin{array}{l}\text { Clinicopathogenetic } \\
\text { features }\end{array}$ & $\begin{array}{c}\text { Aggressive PTC } \\
\text { tissues }(\mathrm{n}=20)\end{array}$ & $\begin{array}{c}\text { Non-aggressive PTC } \\
\text { tissues }(\mathrm{n}=20)\end{array}$ \\
\hline Age (years) & $40.5 \pm 12.7$ & $37.6 \pm 15.2$ \\
Gender (male:female) & $5: 15$ & $3: 17$ \\
Tumor size (cm) & $2.5 \pm 0.8$ & $2.0 \pm 0.3$ \\
Distant metastasis & 3 & 0 \\
Tumor location & & \\
Unilateral & 4 & 6 \\
Bilateral & 16 & 14 \\
TNM staging (AJCC) & & 11 \\
I & 0 & 9 \\
II & 1 & 0 \\
III & 17 & 0 \\
IV & 2 & 12 \\
Multicentricity & 16 & 0 \\
Extrathyroidal & 17 & \\
extension & &
\end{tabular}

PTC, papillary thyroid cancer; AJCC, American Joint Committee on Cancer.

miR-17, miR-199a-5p, miR-342-3p, miR-768-3p and miR-720) and downregulated miRNAs (miR-1278, miR-16-1, miR-613, miR-1225-5p, miR-1268, miR-1826, miR-637, miR-1231, miR-1302 and miR-486-5p) observed in 3 aggressive tumors compared with non-aggressive PTC tissues (Fig. 1).

Validation expression of the miRNAs identified by miRNA array. The microarray data from miRNA expression profiling were validated by qPCR analysis for 12 miRNAs in a cohort of 20 PTC samples with extrathyroidal dissemination and/ or regional lymphatic and distant metastases; and 20 PTC samples with no extrathyroidal dissemination, regional lymphatic or distant metastases (Table I). A significantly altered expression level of 5 miRNAs between aggressive and non-aggressive PTC was observed (Fig. 2). miR-222, miR-221 and miR-146b-5p were upregulated in aggressive PTC, and miR-16 and miR-613 were downregulated in aggressive PTC.

Gene ontology $(G O)$ analysis of $m R N A$ expression assessed by gene expression array. Gene expression profiling was conducted. There was significantly different expression of 2000 genes between aggressive and non-aggressive PTC samples. GO analyses of the differentially expressed genes demonstrated that the expression of cell adhesion genes involved in invasion and metastasis was significantly different in aggressive PTC compared with non-aggressive PTC (Fig. 3).

Identification of target genes for validated miRNAs. To identify the validated miRNA targets, a bioinformatics search (Targetscan, Findtar, miRanda) for putative mRNA targets of the miRNAs was conducted. Among the candidate targets, human TIMP3, ZNFR3, FN1 and ITGA2 contained regions

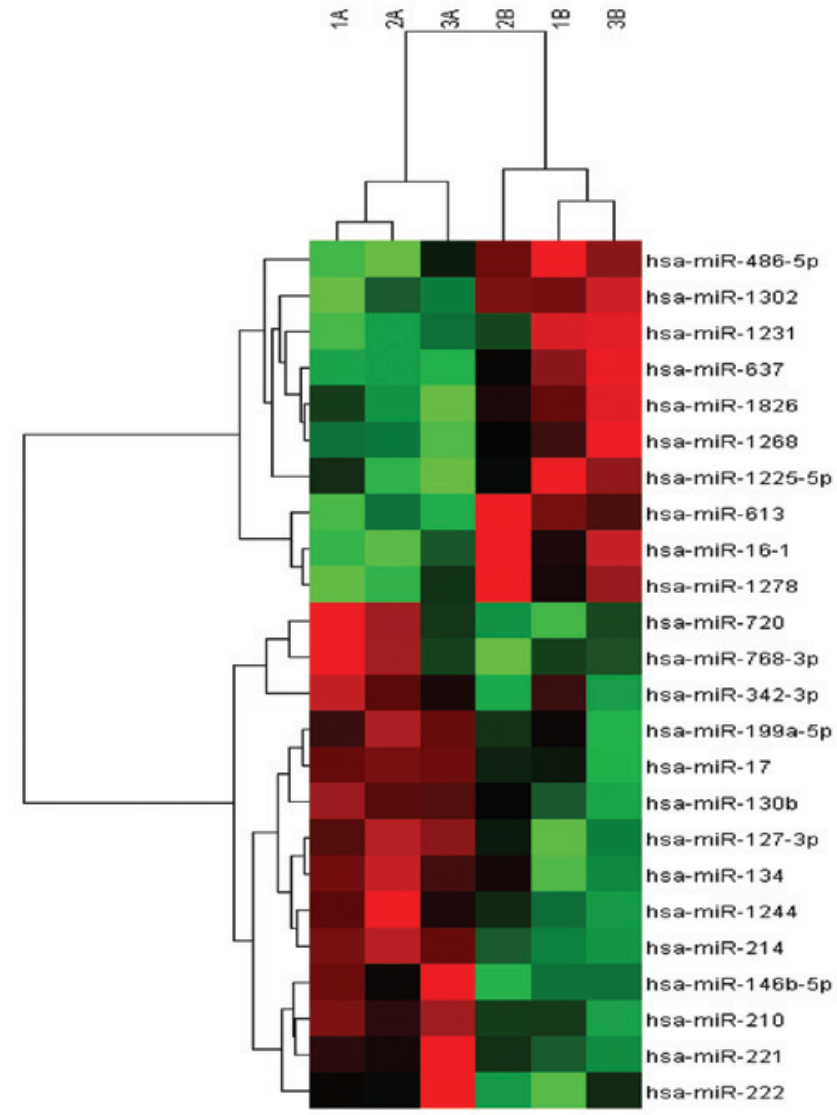

Figure 1. Differentially expressed miRNAs in aggressive $(n=3)$ and paired non-aggressive papillary thyroid cancer (PTC) tissues $(n=3)$.

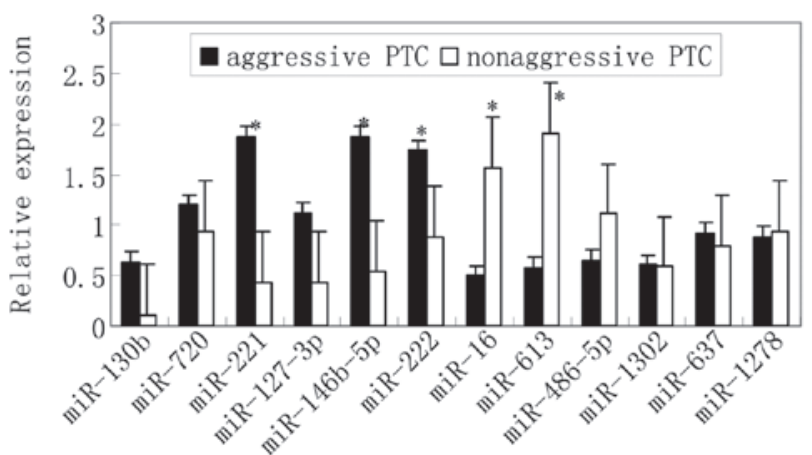

Figure 2. miRNA array data validation by $q P C R$ in aggressive $(n=20)$ and paired non-aggressive papillary thyroid cancer (PTC) tissues $(n=20)$. The fold change values indicate the relative change in the expression levels between samples and the internal control (U6), assuming that the value of the U6 expression level of each sample was equal to 1 . Each bar is the mean $\pm \mathrm{SE}$ from experiments performed in triplicate. ${ }^{*} \mathrm{P}<0.05$.

that matched the seed sequences of $h s a-m i R-221, m i R-222$, $m i R-146 b-5 p, m i R-613$ and $m i R-16$, respectively (Fig. 4). Furthermore, human TIMP3, ZNFR3, FN1 and ITGA2 in gene array were inversely correlated with $h s a-m i R-221, m i R-222$, $m i R-146 b-5 p, m i R-613$ and $m i R-16$ expression in miRNA array between aggressive and non-aggressive PTC. To verify that TIMP3, ZNFR3, FN1 and ITGA2 are direct targets of $m i R-221 / 222, m i R-146 b-5 p, m i R-613$ and $m i R-16$, respectively, a luciferase reporter assay was conducted. The results showed 


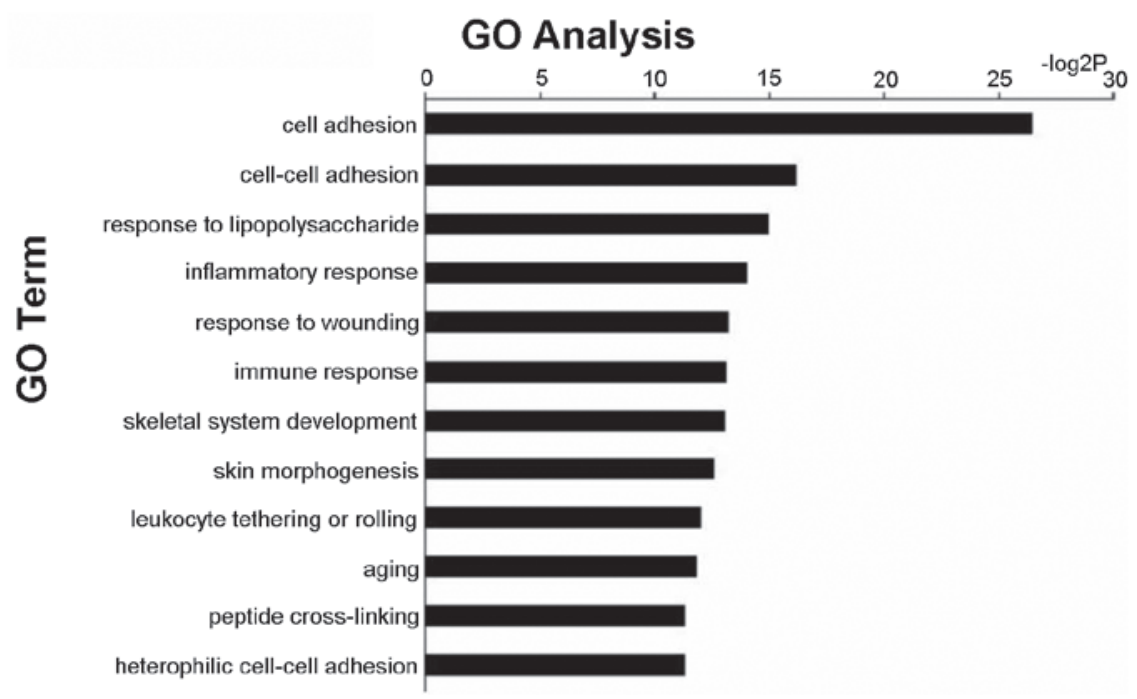

Figure 3. Gene ontology (GO) analysis of differentially expressed genes in aggressive ( $\mathrm{n}=3$ ) and non-aggressive papillary thyroid cancer (PTC) tissues $(\mathrm{n}=3)$.

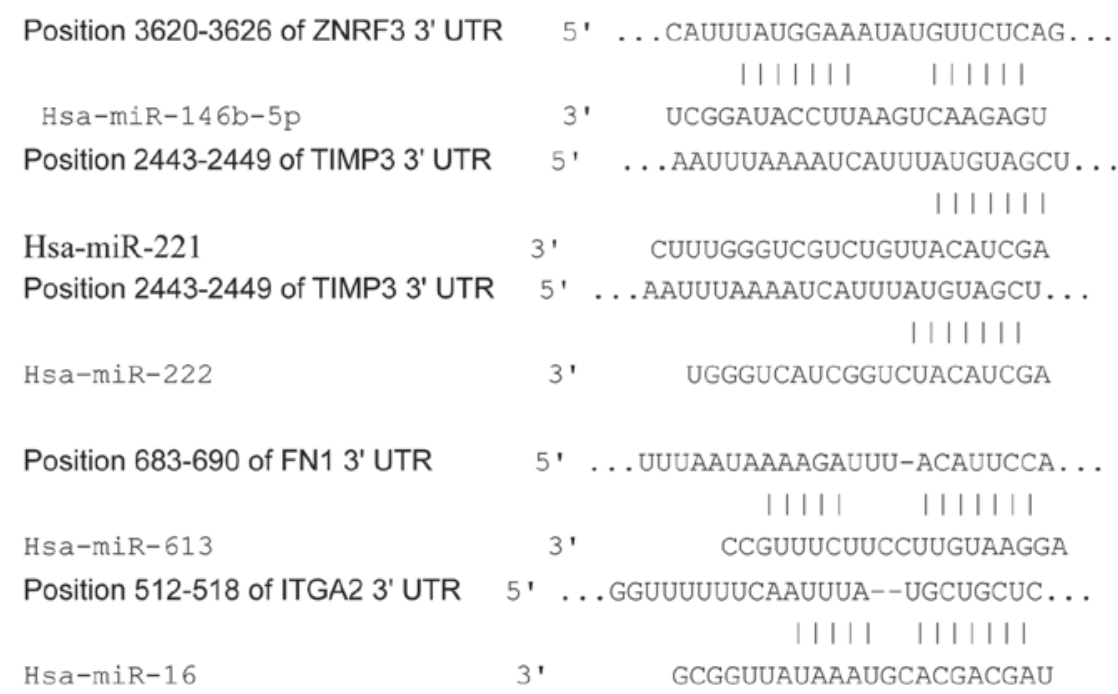

Figure 4. The alignment of the seed regions of the miRNAs with their individual target gene 3'-UTR.

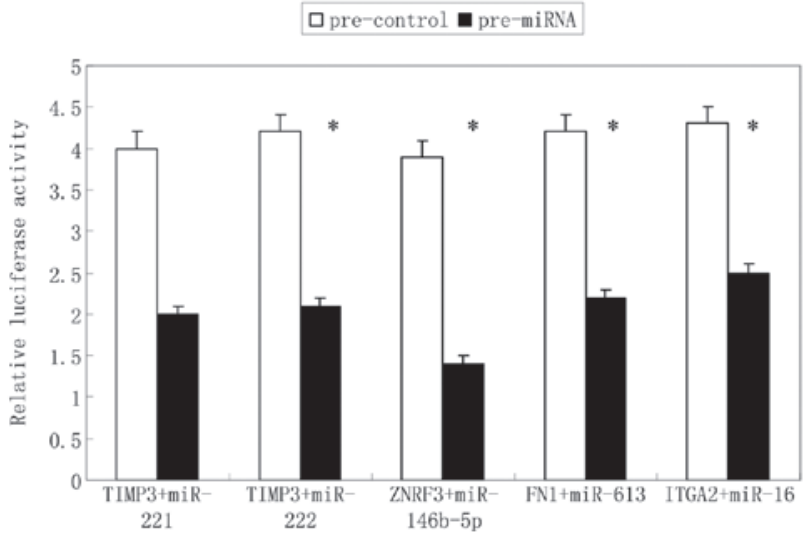

Figure 5. Relative luciferase activity in A549 cells transiently transfected for $24 \mathrm{~h}$ with wild-type constructs for miR-221/222, miR-146b-5p, miR-16 and miR-613 seed sequences and with miR-221/222, miR-146b-5p, miR-16 and miR-613 oligonucleotides and a control non-targeting scrambled oligonucleotide. The relative activity of firefly luciferase expression was standardized to a transfection control, using Renilla luciferase. The bars are the mean $\pm \mathrm{SE}$ $(n=3) .{ }^{*} \mathrm{P}<0.05$ vs. the scrambled oligonucleotide. that transfection of the individual miRNAs significantly affected the upstream luciferase expression of the related gene 3'-UTR (Fig. 5).

Validation expression of the target genes. TIMP3, ZNFR3, FN1 and ITGA2 gene expression validated by qPCR analysis in the same aggressive and non-aggressive PTC tissues as miRNA validation. A significantly altered expression levels of these genes between aggressive and non-aggressive PTC was observed (Fig. 6). The expression of TIMP3 and ZNFR3 genes was downregulated, and the expression of FN1 and ITGA2 genes was upregulated in aggressive PTC, which were negatively correlated with the associated miRNA expression.

\section{Discussion}

In the present study, using the miRNA expression assay as an initial screen, it was demonstrated that a number of miRNAs are differentially expressed in aggressive PTC, characterized 


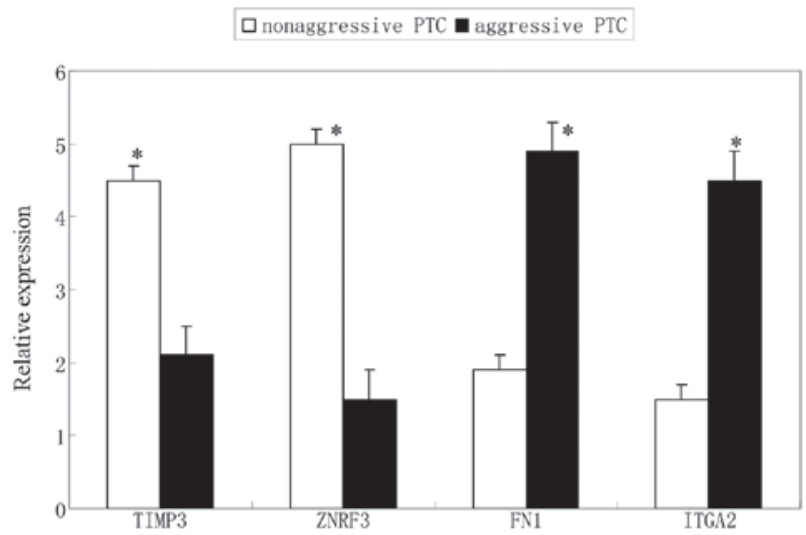

Figure 6. Target gene validation by qPCR. Relative expression values indicate the relative change in TIMP3, ZNFR3, FN1 and ITGA2 mRNA expression levels in aggressive and non-aggressive papillary thyroid cancer (PTC) tissues, normalized to glyceraldehyde 3-phosphate dehydrogenase. The error bars are the mean $\pm \mathrm{SE}(\mathrm{n}=20) .{ }^{*} \mathrm{P}<0.05$.

by extrathyroidal dissemination and/or regional lymphatic and distant metastases, compared with non-aggressive PTC. When expression levels were quantified and validated in a separate comparison cohort, it was observed that aggressive PTCs were characterized by the differential upregulation of miR-221/222 and miR-146b-5p in addition to the downregulation of miR-16 and miR-613. These results suggested a correlation between miR-221/222 and miR-146b-5p upregulation and thyroid carcinoma cell invasion and metastasis. The results also demonstrated that miR-613 and miR-16 may be tumor invasion suppressors in PTC. The observations of miR-221/222 are consistent with numerous previous studies in breast cancer (9), PTC (10), prostate carcinoma (11) and glioma (12). Although studies concerning miR-146b-5p are scarce, Geraldo et al (13) demonstrated in their investigation of a PTC cell line that the overexpression of miR146b-5p rendered cells resistant to TGF- $\beta$-mediated cell-cycle arrest and significantly increased cell proliferation. In the majority of studies concerning the miR-146 subtype is bound to miR-146b. Yip et al (10) and Chou et al (14) demonstrated that miR-146b is highly expressed in PTCs with high-risk features, including extrathyroidal invasion and the $B R A F^{V 600 E}$ mutation. In the most recent study by Chou et al (15) it was demonstrated that overexpression of miR-146b significantly increased cell migration and invasiveness. Furthermore, miR-146b expression was an independent risk factor for poor prognosis in PTC as the BRAF mutation was not associated with poor prognosis. mir-16 is involved in the tumor stem cell apoptosis process (16) and was downregulated in the invasive subpopulations compared with the control subpopulations in a study by Gao et al (17) in human PTC cell lines. To the best of our knowledge, the present study is the first to demonstrate that miR-613 is involved in aggressive PTC and may be tumor suppressive in PTC.

To determine how the deregulated miRNAs affect the aggressive ability of PTC, GO analysis of mRNA expression was assessed with a gene expression array. It was demonstrated that cell adhesion genes involved in invasion and metastasis were differentially expressed in aggressive PTC compared with non-aggressive PTC. To identify the validated miRNA targets, a bioinformatics search (Targetscan, Findtar, MiRanda) for putative mRNA targets of the miRNAs was conducted. It was also shown that TIMP3, ZNFR3, FN1 and ITGA2 are direct targets of miR-221/222, miR-146b-5p, miR-613 and miR-16, respectively, using the luciferase reporter assay. Furthermore, the expression levels of the target genes were quantified and validated by $\mathrm{qPCR}$ in a separate comparison cohort. It was observed that TIMP3 and ZNFR3 gene expression was downregulated with the upregulation of miR-221/222 and miR-146b-5p, while FN1 and ITGA2 gene expression was upregulated with the downregulation of miR-613 and miR-16 in aggressive PTC.

The TIMP3 gene, encoding a metalloproteinase inhibitor was capable of inhibiting growth, angiogenesis, invasion and metastasis of several cancers, and was identified to be silenced by promoter methylation in a consistent fraction of PTCs, in association with tumor aggressiveness and $B R A F^{V 600 E}$ mutation, thus suggesting an oncosuppressive role (18). The cell-surface transmembrane E3 ubiquitin ligase zinc and ring finger 3 was demonstrated to be a negative feedback regulator of Wnt/ $\beta$-catenin signaling (19). Aberrant activation of $\mathrm{Wnt} / \beta$-catenin signaling is involved in the development of several epithelial tumors, including PTCs (20). In addition, it is also identified to be a critical factor in the aggressive phenotype of breast cancer $(21,22)$, colorectal cancer $(23,24)$ and PTCs (25), characterized by malignant features, such as increased cell motility, invasiveness and metastasis (26). Fibronectin is a protein of the extracellular matrix (ECM), whose remodeling and degradation may influence changes in tumor growth and dissemination capacity (27). It is directly involved in cell proliferation and migration, and participates in the ECM changes that occur during physiological and pathological processes via integrin transmembrane receptors, particularly the integrin $\alpha_{5} \beta_{1}$ and $\alpha_{v}$ subtypes $(28,29)$. Integrins are a family of surface receptors that mediate cell-matrix and cell-cell interactions. These receptors consist of $\alpha$ and $\beta$ subunits and each subunit has several isoforms that form at least 25 different integrins $(30,31)$. Integrin $\alpha_{2} \beta_{1}$, also termed platelet glycoprotein Ia-IIa, is expressed by epithelial cells, and its level of expression in tumor cells is associated with motility, invasiveness and cell differentiation (31).

In the present study, the target genes observed are relative to the extracellular matrix or signal transduction pathways concerning tumorigenesis or aggressiveness of tumors. The migration and metastasis of cancer cells occurs in three stages, adherence, degradation and movement (17). Thus, the changes in ECM are pivotal in the aggressive nature of cancer cells. The results of the present study demonstrated that when miR-221/222 and miR-146b-5p were upregulated, the expression of TIMP3 and ZNFR3 were downregulated and when miR-613 and miR-16 downregulated, the expression of FN1 and ITGA2 were upregulated, representing a negative correlation. Thus, the expression levels of the target genes in the present study may lead to the aggressiveness of PTCs, including effects on invasion and migration.

The results of the present study emphasize the importance of miR-221/222, miR-146b-5p, miR-613 and miR-16 in determining the aggressive properties of PTCs and they may exert this function through the TIMP3, ZNFR3, FN1 and ITGA2 genes, respectively. Although further studies are required to 
analyse the correlation, data from the present study provides an insight into the involvement of these miRNAs and their target genes may contribute to the identification of the potential pathway in aggressive PTC.

\section{Acknowledgements}

This study was supported by the National Natural Science Foundation of China (grant no. 81272935).

\section{References}

1. Davies L and Welch HG: Increasing incidence of thyroid cancer in the United States, 1973-2002. JAMA 295: 2164-2167, 2006.

2. Hay ID, Thompson GB, Grant CS, et al: Papillary thyroid carcinoma managed at the Mayo Clinic during six decades (1940-1999): temporal trends in initial therapy and long-term outcome in 2444 consecutively treated patients. World J Surg 26 879-885, 2002

3. Bartel DP: MicroRNAs: target recognition and regulatory functions. Cell 136: 215-233, 2009.

4. White NM, Fatoohi E, Metias M, Jung K, Stephan C and Yousef GM: Metastamirs: a stepping stone towards improved cancer management. Nat Rev Clin Onco 8: 75-84, 2011.

5. Pallante P, Visone R, Croce CM and Fusco A: Deregulation of microRNA expression in follicular-cell-derived human thyroid carcinomas. Endocr Relat Cancer 17: F91-F104, 2010.

6. Wilson C: Cancer: MicroRNA expression provides clues about the aggressiveness of papillary thyroid carcinoma. Nat Rev Endocrinol 6: 416, 2010.

7. Vasko V, Espinosa AV, Scouten W, et al: Gene expression and functional evidence of epithelial-to-mesenchymal transition in papillary thyroid carcinoma invasion. Proc Natl Acad Sci USA 104: 2803-2808, 2007.

8. Yang Z, Fan Y, Deng Z, Wu B and Zheng Q: Amyloid precursor protein as a potential marker of malignancy and prognosis in papillary thyroid carcinoma. Oncol Lett 3: 1227-1230, 2012.

9. Stinson S, Lackner MR, Adai AT, et al: miR-221/222 targeting of trichorhinophalangeal 1 (TRPS1) promotes epithelial-to-mesenchymal transition in breast cancer. Sci Signal 4: 2002258, 2011.

10. Yip L, Kelly L, Shuai Y, et al: MicroRNA signature distinguishes the degree of aggressiveness of papillary thyroid carcinoma. Ann Surg Oncol 18: 2035-2041, 2011.

11. Zheng C, Yinghao S and Li J: MiR-221 expression affects invasion potential of human prostate carcinoma cell lines by targeting DVL2. Med Oncol 29: 815-822, 2012.

12. Zhang C, Zhang J, Hao J, et al: High level of miR-221/222 confers increased cell invasion and poor prognosis in glioma. J Transl Med 10: 119, 2012

13. Geraldo MV, Yamashita AS and Kimura ET: MicroRNA miR-146b-5p regulates signal transduction of TGF- $\beta$ by repressing SMAD4 in thyroid cancer. Oncogene 31: 1910-1922, 2012.
14. Chou CK, Chen RF, Chou FF, et al: $\mathrm{miR}-146 \mathrm{~b}$ is highly expressed in adult papillary thyroid carcinomas with high risk features including extrathyroidal invasion and the $\mathrm{BRAF}^{\mathrm{V} 600 \mathrm{E}}$ mutation. Thyroid 20: 489-494, 2010.

15. Chou CK, Yang KD, Chou FF, et al: Prognostic implications of miR-146b expression and its functional role in papillary thyroid carcinoma. J Clin Endocrinol Metab 98: E196-E205, 2013.

16. Guo CJ, Pan Q, Li DG, Sun H and Liu BW: miR-15b and miR-16 are implicated in activation of the rat hepatic stellate cell: An essential role for apoptosis. J Hepatol 50: 766-778, 2009.

17. Gao Y, Wang C, Shan Z, et al: MiRNA expression in a human papillary thyroid carcinoma cell line varies with invasiveness. Endocr J 57: 81-86, 2010.

18. Anania MC, Sensi M, Radaelli E, et al: TIMP3 regulates migration, invasion and in vivo tumorigenicity of thyroid tumor cells. Oncogene 30: 3011-3023, 2011.

19. Hao HX, Xie Y, Zhang Y, et al: ZNRF3 promotes Wnt receptor turnover in an R-spondin-sensitive manner. Nature 485: 195-200, 2012.

20. Sastre-Perona A and Santisteban P: Role of the Wnt pathway in thyroid cancer. Front Endocrinol (Lausanne) 3: 31, 2012.

21. Ponzo MG, Lesurf R, Petkiewicz S, et al: Met induces mammary tumors with diverse histologies and is associated with poor outcome and human basal breast cancer. Proc Natl Acad Sci USA 106: 12903-12908, 2009.

22. Previdi S, Maroni P, Matteucci E, Broggini M, Bendinelli P and Desiderio MA: Interaction between human-breast cancer metastasis and bone microenvironment through activated hepatocyte growth factor/Met and beta-catenin/Wnt pathways. Eur J Cancer 46: 1679-1691, 2010.

23. Strillacci A, Valerii MC, Sansone P, et al: Loss of miR-101 expression promotes $\mathrm{Wnt} / \beta$-catenin signaling pathway activation and malignancy in colon cancer cells. J Pathol 229: 379-389, 2013.

24. Bandapalli OR, Dihlmann S, Helwa R, et al: Transcriptional activation of the $\beta$-catenin gene at the invasion front of colorectal liver metastases. J Pathol 218: 370-379, 2009.

25. Cho SW, Lee EJ, Kim H, et al: Dickkopf-1 inhibits thyroid cancer cell survival and migration through regulation of $\beta$-catenin/ E-cadherin signaling. Mol Cell Endocrinol 366: 90-98, 2013.

26. Kalluri R and Weinberg RA: The basics of epithelial - mesenchymal transition. J Clin Invest 119: 1420-1428, 2009.

27. Viana Lde S, Affonso RJ Jr, Silva SR, et al: Relationship between the expression of the extracellular matrix genes SPARC, SPP1, FN1, ITGA5 and ITGAV and clinicopathological parameters of tumor progression and colorectal cancer dissemination. Oncology 84: 81-91, 2013.

28. Alexi X, Berditchevski F and Odintsova E: The effect of cell-ECM adhesion on signalling via the ErbB family of growth factor receptors. Biochem Soc Trans 39: 568-573, 2011.

29. Foubert $P$ and Varner JA: Integrins in tumor angiogenesis and lymphangiogenesis. Methods Mol Biol 757: 471-486, 2012.

30. Zhang H, Guo F and Zhu G: Involvement of host cell integrin $\alpha 2$ in Cryptosporidium parvum infection. Infect Immun 80: 1753-1758, 2012.

31. Chen J, Liu NN, Li JQ, et al: Association between ITGA2 C807T polymorphism and gastric cancer risk. World J Gastroenterol 17: 2860-2866, 2011. 